Pacific Journal of Mathematics

COMBINATORIAL GEOMETRY AND ACTIONS OF COMPACT
LIE GROUPS LIE GROUPS 


\section{COMBINATORIAL GEOMETRY AND ACTIONS OF COMPACT LIE GROUPS}

\section{TOR SKJELBRED}

In this paper a theorem of combinatorial geometry will be applied to prove results about actions of compact Lie groups on manifolds.

In order to understand actions on differentiable manifolds, the weights of the tangential representations at fixed points of a maximal torus can be taken as basic data. Those weights are related by the structure of the equivariant cohomology ring of the manifold. The weights can also be considered as just a finite set of vectors or as a finite set of points in a projective space. From this point of view, theorems of combinatorial geometry can be used. Hence representation theory, equivariant cohomology theory, and combinatorial geometry can be used to understand differentiable actions. We will use the following result of combinatorial geometry which has been generalized by Sten Hansen [11]. It was conjectured by Sylvester[16] in 1893 and proved by Gallai in 1933.

THEOREM 1 (Sylvester-Gallai). "Given a finite set of points in the real affine plane, there is a line containing exactly two of those points, unless the point set is collinear."

It was during discussions with Ted Chang concerning his results in equivariant homotopy theory, that I realized that the theorem of Sylvester-Gallai was useful. I am grateful to Ted for explaining Theorem 5 to me. A very simple example of a result in equivariant homotopy theory follows.

THeOREM 2. "Let a torus $T$ be acting on $S^{2 n}$ and on $S^{4 n-1}$, such that the action on $S^{4 n-1}$ is effective and such that $F\left(T, S^{4 n-1}\right)=$ $\varnothing$ and $F\left(T, S^{2 n}\right)$ is connected. If rank $T \geqq 4$, then every equivariant map $S^{4 n-1} \rightarrow S^{2 n}$ has trivial Hopf invariant." The attaching map $S^{15} \rightarrow S^{8}$ of the Cayley projective plane has nontrivial Hopf invariant and admits an effective action of a torus $T$ of rank 3 with $F\left(T, S^{15}\right)=\varnothing$ and $F\left(T, S^{8}\right)=S^{2}$. Hence the bound rank $T \geqq 4$ cannot be relaxed.

In general the results one can prove using combinatorial geometry say that a group acting on a specific manifold with a specific fixed point set cannot have rank exceeding a certain number. An 
example of this kind concerning transitive actions, is the following result which was first proved by Chang-Skjelbred [7].

THEOREM 3. "Let $G$ be a connected compact Lie group and let $H$ be a closed subgroup containing no nontrivial connected normal subgroup of $G$. If $G / H$ is orientable and of Euler characteristic 3, then $\operatorname{rank} G \leqq 4 . "$

The proof we will give here of the theorem does not apply the classification of simple Lie groups, as did the proof in [7].

In combinatorial geometry the theorem of Sylvester-Gallai has passed through stages of reproving [10] and generalization, notably by Motzkin [14] and Sten Hansen [11] who gave the theorem its final form.

THeOREM 4 (Sten Hansen). "Let $\Omega$ be a finite set in real projective space $P_{R}^{n}$ such that $\Omega$ spans $P_{R}^{n}$. Then there are linear hyperplanes $L^{n-2} \subset H^{n-1} \subset P_{R}^{n}$ such that $H^{n-1}$ is spanned by $H^{n-1} \cap$ $\Omega$ and such that there is an $\omega_{0} \in H^{n-1} \cap \Omega$ with

$$
H^{n-1} \cap \Omega=\left(L^{n-2} \cap \Omega\right) \cup\left\{\omega_{0}\right\} . "
$$

Hansen's result is in fact stronger in that it shows how to find a large number of such hyperplanes $L^{n-2} \subset H^{n-1}$. By setting $n=2$, we obtain the theorem of Sylvester-Gallai.

Certain problems concerning transformation groups give rise to problems in combinatorial geometry. The combinatorial feature of transformation groups is apparent in the Golber formulas for torus actions, see $[9,6,4]$. At the end of this paper, we will pose a problem in combinatorial geometry whose solution is of interest in the theory of transformation groups of rank $\geqq 3$.

We will now establish a formal link between combinatorial geometry and the geometry of transformation groups. Let $T$ be a maximal torus of the transformation group, and let $x$ be a fixed point of $T$ in a differentiable manifold $M$ where $T$ is acting differentiably. Let $L(T)$ be the Lie algebra of $T$, and let $L^{*}(T)$ be the set of linear maps $f: L(T) \rightarrow R$ such that $\operatorname{Ker}(f)$ is the Lie algebra of a closed subgroup of $T$. Then $L^{*}(T) \cong \operatorname{Hom}(T, U(1)) \otimes Q$ and $\operatorname{dim}_{Q} L^{*}(T)=\operatorname{rank} T$. The weights of the tangential representation of $T$ at $x \in M$ are elements of $L^{*}(T)$ with certain multiplicities. The weights lie in $\operatorname{Hom}(T, U(1)) \subset L^{*}(T)$, and for this reason we will call them integral weights. The multiplicity of an integral weight $w$ at $x$ is 


$$
\operatorname{mult}_{x}(w)=\frac{1}{2}\left(\operatorname{dim}_{x}(F(\operatorname{Ker} w))-\operatorname{dim}_{x}(F(T)) .\right.
$$

By a weight we will understand an element of the projective space $P\left(L^{*}(T)\right)$. For any weight $\omega$, choose $w \in \omega$ where $w$ is an integral weight, and is nondivisible. We set

$$
\operatorname{mult}_{x}(\omega)=\sum_{n \geq 1} \operatorname{mult}_{x}(n w) \text {. }
$$

The Borel formula (B) at $x$ then follows from the splitting of the tangential representation at $x$ in subspaces of dimension $\leqq 2$. We will also consider topological actions on a space $M$ which is paracompact, connected, and of finite cohomology dimension over $Q$. We will from now on assume either that $M$ is a cohomology manifold over $Q$, or that the ring $H^{*}(M ; Q)$ satisfies Poincaré duality. The latter means that for some $n$, the cup product

$$
H^{i}(M ; Q) \otimes H^{n-i}(M ; Q) \longrightarrow H^{n}(M ; Q) \cong Q
$$

is perfect for all $i$, and we set $\operatorname{dim} M=n$. If $M$ is a cohomology manifold, $\operatorname{dim} M$ is its dimension as such. For certain $M, \operatorname{dim} M$ has now been defined in two ways. One simply has to be consistent, using either local or global cohomology all the time. The cohomology theory will be Čech cohomology with rational coefficients and closed supports. Let a torus $T$ be acting on $M$ and let $F^{1}, F^{2}, \cdots$ be the components of the fixed point set. For any subgroup $K \subset T$, let $F^{i}(K)$ be the component of $F(K, M)$ containing $F^{i}$. If $M$ is a cohomology manifold, then so is each $F^{i}$, according to Conner-Floyd, see [2, p. 81]. If $H^{*}(M ; Q)$ satisfies Poincaré duality, then so does each $H^{*}\left(F^{i} ; Q\right)$, according to the thesis of Ted Chang. Two proofs of this result is given in Chang-Skjelbred [5], and a later third proof appears in Bredon [3]. A fourth proof due to the author appears in [13]. In any case $\operatorname{dim} F^{i}$ is consistently defined. Let $K \subset T$ be a subtorus, that is, a closed connected subgroup of $T$. We set

$$
\operatorname{mult}_{i}(K)=\frac{1}{2}\left(\operatorname{dim} F^{i}(K)-\operatorname{dim} F^{i}\right) .
$$

We say that $K$ is a local weight at $F^{i}$ if $\operatorname{rank} K=\operatorname{rank} T-1$ and $\operatorname{mult}_{i}(K)>0$. Then there is the Borel formula at $F^{i}$,

$$
\operatorname{dim} M-\operatorname{dim} F^{i}=2 \sum \operatorname{mult}_{i}(K)
$$

where the sum extends over all subtori $K$ with $\operatorname{rank} K=\operatorname{rank} T-1$. For cohomology manifolds this formula is due to Borel [2, p. 175], see also Hsiang [12, p. 343]. For Poincaré duality spaces it is due independently to the author and to Allday-Skjelbred [1]. For each 
subtorus $N$ of $T$. there is a Borel formula for the action of $T$ on the invariant subspace $F^{i}(N)$.

$$
\operatorname{dim} F^{i}(N)-\operatorname{dim} F^{i}=2 \sum_{N \subset K} \operatorname{mult}_{i}(K)
$$

where the sum extends over all corank-one subtori $K$ containing $N$, see [12, p. 343]. For each subtorus $K$ of corank-one, $K$ is uniquely determined by the kernel of the restriction map $L^{*}(T) \rightarrow L^{*}(K)$. For any generator $\omega$ of this kernel, we set $K=\omega^{\perp}$. For a general subtorus $N$ of $T$, we set $N=\left(\omega_{1}, \cdots, \omega_{k}\right)^{\perp}$ when $\omega_{1}, \cdots, \omega_{k}$ generate the kernel of $L^{*}(T) \rightarrow L^{*}(N)$. We set $\operatorname{mult}_{i}(\omega)=\operatorname{mult}_{i}\left(\omega^{\perp}\right)$ for each $0 \neq \omega \in L^{*}(T)$, and we say that $\omega$ is a local weight at $F^{i}$ if this multiplicity is positive. For any $0 \neq a \in Q$ we consider $\omega$ and $a \omega$ as identical local weights since $\omega^{\llcorner}=(a \omega)^{\perp}$. The Borel formula can then be written

$$
\begin{aligned}
& \operatorname{dim} M-\operatorname{dim} F^{i}=2 \sum_{\omega} \operatorname{mult}_{i}(\omega) \\
& \operatorname{dim} F^{i}(N)-\operatorname{dim} F^{i}=2 \sum_{\omega\left(L^{*}(N)\right)=0} \operatorname{mult}_{i}(\omega)
\end{aligned}
$$

where the latter sum extends over all $\omega$ such that $N \subset \omega^{\perp}$. If $N=$ $\left(\omega_{1}, \cdots, \omega_{k}\right)^{\perp}$, then $N \subset \omega^{\perp}$ if and only if $\omega$ is a linear combination of $\omega_{1}, \cdots, \omega_{k}$. An " $F$-variety containing $F^{i}$ " is a set of the form $F^{i}(N)$ where $N=\left(\omega_{1}, \cdots, \omega_{k}\right)^{\perp}$ and each $\omega_{i}$ is a local weight of $F^{i}$. Now if $F^{i}(N)$ contains $F^{j}$ as a set, it is also true that $F^{i}(N)=$ $F^{j}(N)$ is an " $F$-variety containing $F^{j}$ ". Hence, there are local weights $\lambda_{1}, \cdots, \lambda_{p}$ at $F^{j}$ such that

$$
\left(\lambda_{1}, \cdots, \lambda_{p}\right)^{\perp}=\left(\omega_{1}, \cdots, \omega_{k}\right)^{\perp}=N .
$$

For a proof of this result in the case where $H^{*}(M ; Q)$ satisfies Poincaré duality, we refer to Chang-Skjelbred [7]. We say that $F^{i}(N)$ is a corank-a $F$-variety where $a=\operatorname{corank} N$. We say that the action of $T$ on $M$ is $c$-effective if for each circle subgroup $S$ of $T$, and each component $Y$ of $F(S, M), \operatorname{dim} Y<\operatorname{dim} M$ or $F(S, M)=\varnothing$. When $M$ is a cohomology manifold, this means that the ineffective kernel of the action is finite. When $H^{*}(M ; Q)$ satisfies Poincare duality, an elaboration of the concept of $c$-effectiveness is found in [7]. In both cases, if $F(T, M) \neq \varnothing$, the action is c-effective if and only if the local weights at each $F^{i}$ generate $L^{*}(T)$.

Theorem 5 (Ted Chang). "Let a torus $T$ of rank $\geqq 2$ be acting c-effectively on $M$, and let $F^{1}$ and $F^{2}$ be two components of the fixed point set. Assume that every corank-one F-variety containing $F^{1}$ also contains $F^{2}$. Let $\Omega=\left\{\omega_{1}, \omega_{2}, \cdots\right\}$ be the set of local weights at $F^{1}$ and let $N=\left\{\nu_{1}, \nu_{2}, \cdots\right\}$ be the set of local weights at $F^{2}$ which 
are not local weights at $F^{1}$. Then $\operatorname{dim} F^{2} \geqq \operatorname{dim} F^{1}$, and if $\operatorname{dim} F^{2}>\operatorname{dim} F^{1},(N, \Omega)$ satisfies the following properties (i) and (ii). Here Lin( ) will denote linear span over $Q$.

(i) For each pair $\omega_{i}, \omega_{j} \in \Omega$, there is some $\nu_{k} \in N$ such that $\operatorname{Lin}\left(\omega_{\imath}, \omega_{j}\right)=\operatorname{Lin}\left(\omega_{i}, \nu_{k}\right)$.

(ii) For each pair $\omega_{i} \in \Omega, \nu_{k} \in N$, there is some $\omega_{j} \in \Omega$ such that $\operatorname{Lin}\left(\omega_{i}, \nu_{k}\right)=\operatorname{Lin}\left(\omega_{i}, \omega_{j}\right) . "$

Proof. Since each $F^{1}\left(\omega_{\imath}^{1}\right)$ contains $F^{2}$, it follows that each $\omega_{i}$ is a local weight at $F^{2}$. Since $F^{1}\left(\omega_{i}^{\frac{1}{1}}\right)=F^{2}\left(\omega_{i}^{!}\right)$, we have

$$
\begin{aligned}
& 2 \operatorname{mult}_{1}\left(\omega_{\imath}\right)-2 \operatorname{mult}_{2}\left(\omega_{i}\right) \\
& \quad=\left(\operatorname{dim} F^{1}\left(\omega_{i}^{\perp}\right)-\operatorname{dim} F^{1}\right)-\left(\operatorname{dim} F^{2}\left(\omega_{i}^{\perp}\right)-\operatorname{dim} F^{2}\right) \\
& \quad=\operatorname{dim} F^{2}-\operatorname{dim} F^{1}, \text { for all } \omega_{i} .
\end{aligned}
$$

Let $\omega_{i}, \omega_{j} \in \Omega$. The Borel formulas of $X=F^{1}\left(\left(\omega_{i}, \omega_{j}\right)^{\perp}\right)=F^{2}\left(\left(\omega_{\imath}, \omega_{j}\right)^{\perp}\right)$ at $F^{1}$ and at $F^{2}$ are as follows, where $V=\operatorname{Lin}\left(\omega_{2}, \omega_{j}\right)$.

$$
\begin{aligned}
& \operatorname{dim} X-\operatorname{dim} F^{1}=2 \sum_{\omega_{k} \in V} \operatorname{mult}_{1}\left(\omega_{k}\right), \\
& \operatorname{dim} X-\operatorname{dim} F^{2}=2 \sum_{\omega_{\omega_{k} \in V}} \operatorname{mult}_{2}\left(\omega_{l_{k}}\right)+2 \sum_{\nu_{p} \in V} \operatorname{mult}_{2}\left(\nu_{p}\right) .
\end{aligned}
$$

Subtracting, we obtain

$$
\operatorname{dim} F^{2}-\operatorname{dim} F^{1}=\sum_{\omega_{k} \in V}\left(\operatorname{dim} F^{2}-\operatorname{dim} F^{1}\right)-2 \sum_{\nu_{p} \in V} \operatorname{mult}_{2}\left(\nu_{p}\right) .
$$

Since $\omega_{k} \in V$ for $k=i, j$, we obtain

$$
2 \sum_{\nu_{p} \in V} \operatorname{mult}_{2}\left(\nu_{p}\right) \geqq \operatorname{dim} F^{2}-\operatorname{dim} F^{1} \geqq 0 .
$$

In case $\operatorname{dim} F^{2}>\operatorname{dim} F^{1}$, there must be some $\nu_{p} \in V=\operatorname{Lin}\left(\omega_{\imath}, \omega_{j}\right)$ so that property (i) holds. For property (ii), let $\omega_{i} \in \Omega$ and $\nu_{k} \in N$ be arbitrary. We then have

$$
F^{1} \cup F^{2} \subset F^{1}\left(\omega_{i}^{\perp}\right) \subset F^{1}\left(\left(\omega_{i}, \nu_{k}\right)^{\perp}\right)=Y \text {, say. }
$$

The Borel formula for $Y$ at $F^{1}$ is, with $W=\operatorname{Lin}\left(\omega_{i}, \nu_{k}\right)$,

$$
\operatorname{dim} Y-\operatorname{dim} F^{1}=2 \sum_{\omega_{s} \in W} \operatorname{mult}_{1}\left(\omega_{s}\right) .
$$

And at $F^{2}, \operatorname{dim} Y-\operatorname{dim} F^{2}$

$$
\begin{aligned}
& =2 \sum_{\omega_{s} \in W} \operatorname{mult}_{2}\left(\omega_{s}\right)+2 \sum_{\nu_{p} \in W} \operatorname{mult}_{2}\left(\nu_{p}\right) \\
& \geqq 2 \operatorname{mult}_{2}\left(\omega_{\imath}\right)+2 \operatorname{mult}_{2}\left(\nu_{k}\right),
\end{aligned}
$$

and hence

$$
\begin{aligned}
\operatorname{dim} Y & \geqq \operatorname{dim} F^{2}+2 \operatorname{mult}_{2}\left(\omega_{i}\right)+2 \operatorname{mult}_{2}\left(\boldsymbol{\nu}_{k}\right) \\
& =\operatorname{dim} F^{1}+2 \operatorname{mult}_{1}\left(\omega_{i}\right)+2 \operatorname{mult}_{2}\left(\boldsymbol{\nu}_{k}\right) .
\end{aligned}
$$


Inserting this in the formula at $F^{1}$, we obtain

$$
\sum_{\omega_{s} \in W} \operatorname{mult}_{1}\left(\omega_{s}\right) \geqq \operatorname{mult}_{1}\left(\omega_{i}\right)+\operatorname{mult}_{2}\left(\nu_{k}\right)>\operatorname{mult}_{1}\left(\omega_{i}\right),
$$

and hence there is some $\omega_{j} \in W=\operatorname{Lin}\left(\omega_{i}, \nu_{k}\right), \omega_{j} \neq \omega_{i}$, and (ii) holds.

Lemma 6. "Let $T$ be a torus acting on $M$ where $H^{*}(M ; Q)$ satisfies Poincaré duality, such that the fixed point set has two components $F=F^{1} \cup F^{2}, \operatorname{dim}_{Q} H^{*}(M ; Q)=\operatorname{dim}_{Q} H^{*}(F ; Q)$ and where $H^{*}(M ; Q) \rightarrow H^{*}\left(F^{1} ; Q\right)$ is onto. Then each corank-one F-variety containing $F^{1}$ also contains $F^{2}$ and hence Theorem 5 applies."

Proof. If there were a $F$-variety $F^{1}(K)$ not containing $F^{2}$, then by equivariant cohomology theory, $\operatorname{dim}_{Q} H^{*}\left(F^{i}(K) ; Q\right)=$ $\operatorname{dim}_{Q} H^{*}\left(F^{i} ; Q\right), i=1,2$.

As $H^{*}\left(F^{1}(K) ; Q\right) \rightarrow H^{*}\left(F^{1} ; Q\right)$ is onto, it is an isomorphism. When corank $K=1$, we then have $\operatorname{mult}_{1}(K)=0$, so that $K$ is not a local weight at $F^{1}$ and $F^{1}(K)$ is not a corank-one $F$-variety at $F^{1}$. (Here $\operatorname{dim} F^{1}(K)=\operatorname{dim} F^{1}$ because "dim" is taken in the Poincaré duality sense.) This contradiction concludes the proof.

We now come to the connection with combinatorial geometry. When a torus of rank $n$ is acting, the sets $N$ and $\Omega$ of Theorem 5 are strictly speaking sets of points in $P_{Q}^{n-1}$.

Definition 7. "Given two finite disjoint sets $N$ and $\Omega$ of points in real projective space $P_{R}^{m}$, we say that $(N, \Omega)$ is a special figure in $P_{R}^{m}$ if $\Omega$ spans $P_{R}^{m}$ and the properties (i) and (ii) of Theorem 5 hold, that is, letting $L()$ denote the join of linear subspaces,

(i) For each pair of points $\omega, \omega^{\prime}$ in $\Omega$, there is some $\nu \in N$ with $\nu \in L\left(\omega, \omega^{\prime}\right)$.

(ii) For each pair of points $\omega \in \Omega, \nu \in N$, there is some $\omega^{\prime} \in \Omega$ with $\omega^{\prime} \in L(\omega, \nu)$ and $\omega^{\prime} \neq \omega . "$

THEOREM 8. "There is a special figure in $P_{R}^{m}$ only when $m \leqq 2 . "$

Proof. Assume $m \geqq 3$ and let $L^{m-2} \subset H^{m-1} \subset P_{R}^{m}$ be the hyperplanes whose existence is guaranteed by the theorem of Sten Hansen. Then $H^{m-1}$ is generated by $\Omega \cap H^{m-1}$ and there is $\omega_{0} \in \Omega$ such that

$$
\Omega \cap H^{m-1}=\left(\Omega \cap L^{m-2}\right) \cup\left\{\omega_{0}\right\} .
$$

Then $\Omega \cap L^{m-2}$ generates $L^{m-2}$ and since $m-2 \geqq 1$, there are at 
least two points $\omega_{1}, \omega_{2} \in \Omega \cap L^{m-2}$. By property (i), there is some $\nu \in N \cap L\left(\omega_{1}, \omega_{2}\right)$, and by property (ii) there is some $\omega_{3} \neq \omega_{0}$ with $\omega_{3} \in \Omega \cap L\left(\omega_{0}, \nu\right)$. Clearly $\omega_{3} \in \Omega \cap H^{m-1}$ and since $\omega_{3} \neq \omega_{0}$, we have $\omega_{3} \in \Omega \cap L^{m-2}$. Hence, $\omega_{3} \in L^{m-2} \cap L\left(\omega_{0}, \nu\right)=\{\nu\}$ and this shows that $\Omega \cap N \neq \varnothing$, a contradiction.

THEOREM 9. "Let a torus $T$ be acting c-effectively on $M$ such that the assumptions of Lemma 6 are satisfied. Then rank $T \leqq 3$."

Proof. Since Theorem 5 applies to the sets $N, \Omega$ of local weights at $F^{1}$ and $F^{2}$, they satisfy properties (i) and (ii) of that theorem. Since the action is c-effective, $L^{*}(T)$ is generated by $\Omega$. Hence $(N, \Omega)$ is a special figure in $P_{Q}^{n-1}$ where $n=\operatorname{rank} T$.

By Theorem $8, n-1 \leqq 2$ and hence $\operatorname{rank} T \leqq 3$.

Corollary. A proof of Theorem 2. Assume that the map $f: S^{4 n-1} \rightarrow S^{2 n}$ has nontrivial Hopf invariant. Let $C_{f}$ be the mapping cone of $f$, with vertex $v$. Then the fixed point set of $T$ in $C_{f}$ is $\{v\} \cup F\left(T, S^{2 n}\right)$. Since $F\left(T, S^{2 n}\right)$ is connected by assumption, and $H^{*}\left(C_{f} ; Q\right)$ satisfies Poincaré duality, the assumptions of Lemma 6 are satisfied by this action. By theorem 9 , rank $T \leqq 3$. Hence, if rank $T \geqq 4$, the Hopf invariant of $f$ must be trivial.

Proof of Theorem 3. Here $G / H$ is orientable of Euler characteristic 3. Let $W H, W G$ be the Weyl group of $H, G$. We now use a result of [7], that $W H$ is the normalizer of $W H^{0}$ in $W G$. This result is shown there in a classification free way. Let $T \subset H$ be a maximal torus; it has fixed point set $F(T, G / H)=N(T) H / H=$ $W G / W H$ consisting of three points. The tangential representation of $T$ at a fixed point $g H \in G / H, g \in N(T)$, has weights $\Delta(G)-$ $\Delta\left(g H^{0} g^{-1}\right)$ where $\Delta G$ is the root system of $G$. If for all $g \in N(T)$, $\Delta\left(H^{0}\right)=\Delta\left(g H^{0} g^{-1}\right)$, then $N(T)$ would normalize $H^{0}, W G$ would normalize $W H^{\circ}$, and hence $W H=W G$, a contradiction. Let $\alpha$ be a root of $H^{0}$ which is not a root of $g H^{0} g^{-1}$ for some $g \in N(T)$. Then the point $p=1 H$ is a component of $Y=F\left(\alpha^{\perp}, G / H\right)$, and $Y$ must have a component of dimension 2 since $\alpha$ is a local weight of multiplicity one at the point $g H$. By equivariant cohomology theory, $Y=\{p\} \cup S^{2}$. Since $\alpha^{\perp}$ is acting effectively on $G / H$ with fixed point set $Y$, it follows from Theorem 9 that $\operatorname{rank} \alpha^{\perp} \leqq 3$, and hence that $\operatorname{rank} G=\operatorname{rank} T \leqq 4$.

A problem in combinatorial geometry. This is the problem of finding all special figures in $P_{Q}^{2}$ and in $P_{R}^{2}$, see Definition 7 . The special figure arising from torus actions such as in Chang's Theo- 
rem 5, all lie in $P_{Q}^{2}$. So far only one type of special figure in $P_{Q}^{2}$ is known; this is the figure arising from the action of $\alpha^{\perp}$ on $G / H$ in the proof of Theorem 3 in case $\operatorname{rank} G=4$. There are such actions on the Cayley projective plane. According to Bredon, a regular pentagon gives rise to a special figure in $P_{R}^{2}$ which has card $\Omega=6$, card $N=10$. In the drawings, the points of $\Omega$ are marked by dots, and the remaining intersection points of two lines are points of $N$. The figure with $P_{i}$ and $Q_{i}$ is a centered pentagon if the lines $P_{i} Q_{i}$ intersect in a common point which we call the centre of $P_{1} P_{2} P_{3} P_{4} P_{5}$. Applying Pappus' theorem to the hexagons of the form $Q_{2} Q_{5} P_{5} Q_{4} Q_{1} P_{1}$, B. Elstad has shown that a centered pentagon determines a special figure just as does a regular pentagon. For this special figure, $\Omega=\left\{P_{1}, P_{2}, P_{3}, P_{4}, P_{5}, C\right\}$ where $C$ is the centre of the pentagon. Elstad and W. Fenchel have both shown that a centered pentagon cannot have rational coordinates of all $P_{i}$. Elstad [8] has shown that choosing four points $P_{i}, i \leqq 4$, with

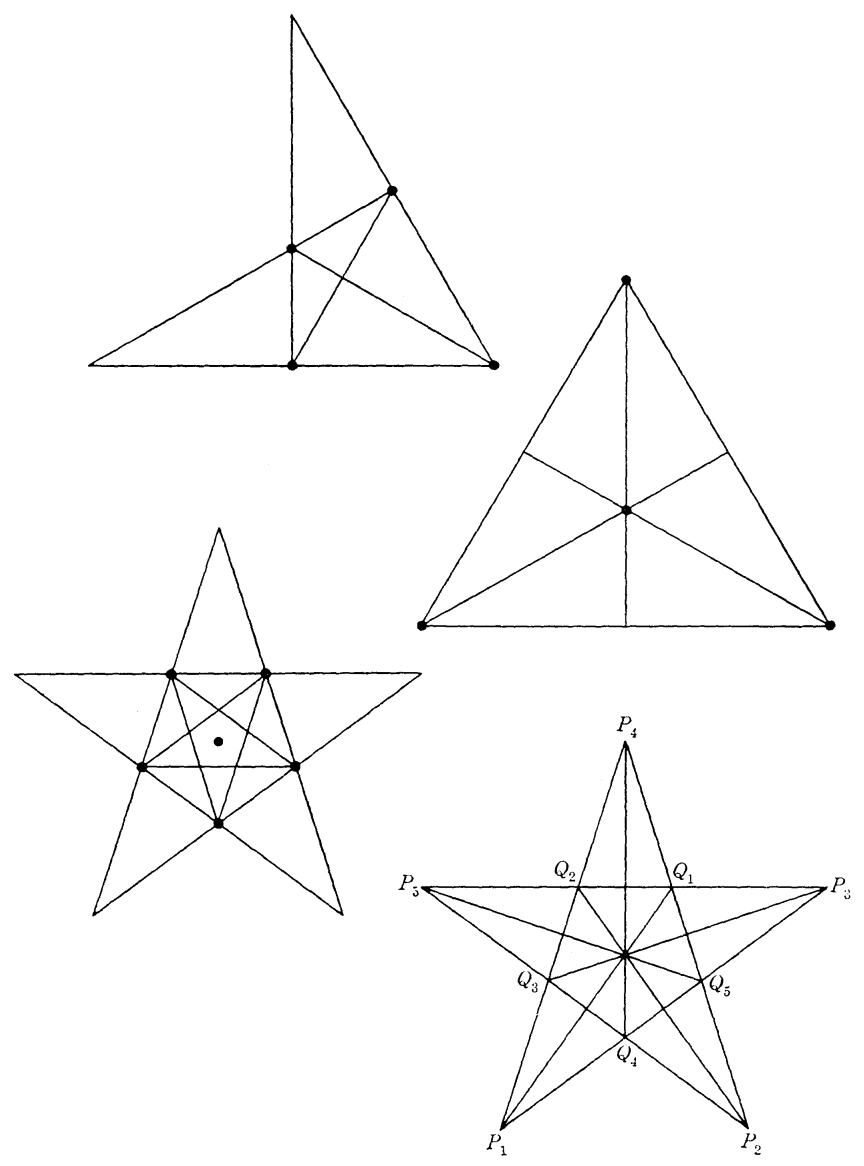


rational coordinates, no three of which are collinear, there are two choices of $P_{5}$ giving a centered pentagon, and that the coordinates of $P_{5}$ are not rational but lie in $Q(\sqrt{5})$.

\section{Problems. (1) Show that for any special figure in $P_{Q}^{2}$, card $\Omega=4$.}

(2) Show that for any special figure in $P_{R}^{2}$, card $\Omega=6$ or 4 .

\section{REFERENCES}

1. C. Allday and T. Skjelbred, The Borel formula for torus actions on a Poincaré duality space, Ann. of Math., 100 (1974), 322-325.

2. A. Borel et. al., Seminar on Transformation Groups, Ann. of Math. Studies, No. 46, Princeton U. Press, Princeton, 1960.

3. G. Bredon, Fixed point sets of actions on Poincaré duality spaces, Topology, 12 (1973), 159-175.

4. - The free part of a torus action and related numerical equalities, Duke Math. J., 41 (1974), 843-854.

5. T. Chang and T. Skjelbred, Group actions on Poincaré duality spaces, Bull. Amer. Math. Soc., 78 (1972), 1024-1026.

6. The topological Schur lemma and related results, Ann. of Math., 100 (1974), 307-321.

7. - Lie group actions on a Cayley projective plane and orientable homogeneous spaces of prime Euler characteristic, Amer. J. Math., 98 (1976), 655-678.

8. B. Elstad, unpublished note.

9. D. Golber, Torus actions on a product of two odd spheres, Topology, 10 (1971), 313-326.

10. Hadwiger und Debrunner, Kombinatorische Geometrie der Ebene.

11. S. Hansen, A generalization of a theorem of Sylvester, Math. Scand., 16 (1965), $175-180$.

12. W. Y. Hsiang, On the Splitting Principle and the Geometric Weight System of Topological Transformation Groups, I, Springer Lecture Notes in Mathematics, 298 (1972), 334-402.

13. Cohomology Theory of Topological Transformation Groups, Ergebnisse der Mathematik Band 85, Springer Verlag, 1975.

14. T. Motzkin, The lines and planes connecting the points of a finite set, Trans. Amer. Math. Soc., 70 (1951), 451-464.

15. T. Skjelbred, Torus actions on manifolds and affine dependence relations, Mimeographed, 1975, IHES, 91 Bures-sur-Yvette, France.

16. Sylvester, Educational Times, 59 (1893), 98.

Received September 1, 1977.

UNIVERSITY OF OSLO

MATEMATISK INSTITUTT

Blindern, Oslo 3, Norway 



\section{PACIFIC JOURNAL OF MATHEMATICS}

EDITORS

RICHARD ARENS (Managing Editor)

University of California

Los Angeles, California 90024

C. W. Curtis

University of Oregon

Eugene, OR 97403

C. C. MOORE

University of California

Berkeley, CA 94720
J. DUGUNDJI

Department of Mathematics University of Southern Californı Los Angeles, California 90007

R. Finn and J. Milgram Stanford University Stanford, California 94305

\section{ASSOCIATE EDITORS}

E. F. BECKENBACH

B. H. NEUMANN

F. WOLF

K. YoSHIDA

\section{SUPPORTING INSTITUTIONS}

UNIVERSITY OF BRITISH COLUMBIA
CALIFORNIA INSTITUTE OF TECHNOLOGY
UNIVERSITY OF CALIFORNIA
MONTANA STATE UNIVERSITY
UNIVERSITY OF NEVADA, RENO
NEW MEXICO STATE UNIVERSITY
OREGON STATE UNIVERSITY
UNIVERSITY OF OREGON

UNIVERSITY OF SOUTHERNEALIFORNIA STANFORD UNIVERSITY UNIVERSITY OF HAWAII UNIVERSITY OF TOKYO UNIVERSITY OF UTAH WASHINGTON STATE UNIVERSITY UNIVERSITY OF WASHINGTON 


\section{Pacific Journal of Mathematics \\ Vol. 79 , No. 1 \\ May, 1978}

Teófilo Abuabara, A remark on infinitely nuclearly differentiable

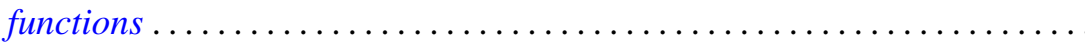

David Fenimore Anderson, Projective modules over subrings of $k[X, Y]$

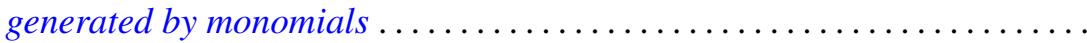

Joseph Barback and Thomas Graham McLaughlin, On the intersection of

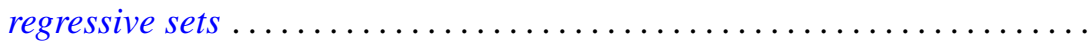

Murray Bell, John Norman Ginsburg and R. Grant Woods, Cardinal inequalities for topological spaces involving the weak Lindelof

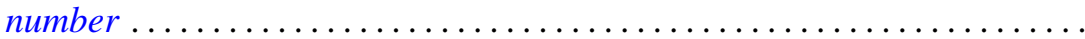

Laurence Richard Boxer, The space of ANRs of a closed surface ............

Zvonko Cerin, Homotopy properties of locally compact spaces at

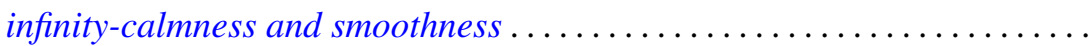

Isidor Fleischer and Ivo G. Rosenberg, The Galois connection between partial functions and relations..................................

John R. Giles, David Allan Gregory and Brailey Sims, Geometrical implications of upper semi-continuity of the duality mapping on a Banach

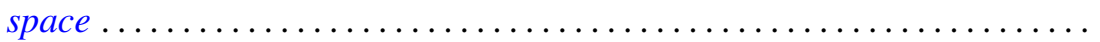

Troy Lee Hicks, Fixed-point theorems in locally convex spaces ............ Hugo Junghenn, Almost periodic functions on semidirect products of transformation semigroups ........................

Victor Kaftal, On the theory of compact operators in von Neumann algebras. II . . . .

Haynes Miller, A spectral sequence for the homology of an infinite

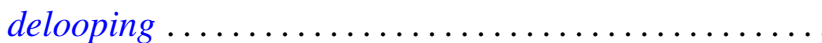

Sanford S. Miller, Petru T. Mocanu and Maxwell O. Reade, Starlike integral operators...

Stanley Stephen Page, Regular FPF rings ...............

Ghan Shyam Pandey, Multipliers for C, 1 summability of Fourier series ...

Shigeo Segawa, Bounded analytic functions on unbounded covering surfaces...

Steven Eugene Shreve, Probability measures and the C-sets of

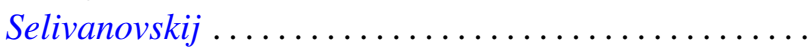

Tor Skjelbred, Combinatorial geometry and actions of compact Lie groups....

Alan Sloan, A note on exponentials of distributions.

Colin Eric Sutherland, Type analysis of the regular representation of a nonunimodular group.

Mark Phillip Thomas, Algebra homomorphisms and the functional

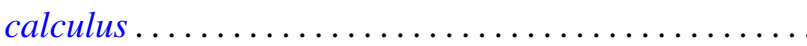

Sergio Eduardo Zarantonello, A representation of $H^{p}$-functions with

$0<p<\infty$. 